\title{
Emergência de Relações Numéricas sob Controle Condicional em Crianças Surdas ${ }^{1}$
}

\author{
Ruth Daisy Capistrano de Souza \\ Grauben José Alves de Assis² \\ Universidade Federal do Pará
}

\begin{abstract}
RESUMO - Os estudos sobre classes ordinais têm apresentado diversos achados experimentais para a compreensão das relações entre estímulos em sequiências. O objetivo do presente trabalho foi investigar se esses resultados seriam obtidos em crianças com surdez. Um procedimento de treino por encadeamento envolveu três classes de estímulos: A = nomes impressos dos números, $\mathrm{B}=$ numerais em língua brasileira de sinais e, $\mathrm{C}=$ formas abstratas, com valores de 1 a 6 . O participante deveria responder na presença da cor vermelha a seqüência $\mathrm{A} 1 \rightarrow \mathrm{A} 2 \rightarrow \mathrm{A} 3 \rightarrow \mathrm{A} 4 \rightarrow \mathrm{A} 5 \rightarrow \mathrm{A} 6$ e na presença da cor verde: A6 $\rightarrow \mathrm{A} 5 \rightarrow \mathrm{A} 4 \rightarrow \mathrm{A} 3 \rightarrow \mathrm{A} 2 \rightarrow$ A1. Após responder corretamente cada seqüência, uma animação gráfica era apresentada na tela. Após revisão da linha de base, testes eram aplicados para avaliar transitividade e conectividade na emergência de classes ordinais. Os resultados mostraram que os participantes responderam prontamente. Conclui-se que o procedimento é eficiente na formação de comportamentos conceituais numéricos e que os estímulos eram funcionalmente equivalentes.
\end{abstract}

Palavras-chave: equivalência de estímulos; classes ordinais; relações numéricas; crianças surdas.

\section{Numeric Relations Emergency Under Conditional Control in Deaf Children}

\begin{abstract}
Studies focusing on the formation of ordinal classes have reported a number of experimental findings on the comprehension of sequential stimulus relations. The aim of this investigation was to replicate previous findings in deaf children. A procedure was used to chain responses to three stimuli classes: $\mathrm{A}=$ printed number names, $\mathrm{B}=$ numbers presented in Brazilian Sign Language and $\mathrm{C}=$ abstract forms in different quantities. The values varied from 1 to 6 . Each participant responded in the presence of green-colored stimuli, presented in a $\mathrm{A} 1 \rightarrow \mathrm{A} 2 \rightarrow \mathrm{A} 3 \rightarrow \mathrm{A} 4 \rightarrow \mathrm{A} 5 \rightarrow \mathrm{A} 6$ sequence; then to red-colored stimuli: in the $\mathrm{A} 6 \rightarrow \mathrm{A} 5 \rightarrow \mathrm{A} 4 \rightarrow \mathrm{A} 3 \rightarrow \mathrm{A} 2 \rightarrow \mathrm{A} 1$ sequence. After having responded correctly to each sequence, an animated figure appeared on the screen. After a revised baseline for each response sequence, tests of transitivity and connectivity were administered in order to evaluate the emergence of ordinal classes. The results showed that the participants responded quickly. In conclusion, it can be said that the procedure was efficient in facilitating the acquisition of numerical concepts, and showed the emergence of equivalent stimulus classes.
\end{abstract}

Key words: stimulus equivalence; ordinal classes; numerical relations; deaf children.

Sidman e Tailby (1982) propuseram que uma classe de equivalência é definida a partir das seguintes propriedades matemáticas, com base na da teoria dos conjuntos: reflexividade, simetria e transitividade. Na reflexividade, um estímulo está relacionado a ele mesmo. Em um teste de reflexividade apresenta-se um estímulo como modelo e dois ou mais estímulos de comparação, um deles semelhante ao modelo, e pede-se ao participante que aponte, dentre as comparações disponíveis, qual o que vai com o modelo. Na simetria, há a mudança das funções de estímulo modelo e de comparação. Assim, se um dado estímulo $a$ relaciona-se com um estímulo $b$, a relação inversa é verdadeira, ou seja, $b$ também se relaciona $\operatorname{com} a$. Portanto, teremos uma reversibilidade funcional.

1 Trabalho baseado na Dissertação de Mestrado do Primeiro autor, sob orientação do segundo. Os autores agradecem o apoio da Unidade de Ensino Especializado Prof. Astério de Campos (Seduc-PA). Os autores agradecem o apoio da bolsista de Iniciação Científica, Mariana Morais Miccione na coleta de dados e ao programador João Vicente do Nascimento pelo desenvolvimento do software.

2 Endereço: Travessa Castelo Branco, 1923/301, Bairro do Guamá, Belém, PA, Brasil 66063-420.E-mail: gjaa@cpgp.ufba.br
Na transitividade, uma nova relação é formada a partir de duas anteriores nas quais há um estímulo comum. Por exemplo, se $a$ se relaciona $\operatorname{com} b$ e este se relaciona $\operatorname{com} c$, então se pode afirmar que $a$ e $c$ se relacionam. À situação inversa, isto é, $c$ relaciona-se com $a$, Sidman e Tailby chamaram de relação simétrica da transitividade e a consideraram como o teste final para verificar a formação de classes de estímulos equivalentes. O paradigma de equivalência por analisar a emergência de classes de estímulos, tem contribuído para a compreensão da aquisição da linguagem, produzindo vários estudos, inclusive em pessoas com repertório verbal mínimo (Carr, Wilkinson, Blackman \& McIlvane, 2000) e com retardo mental (Yamamoto, 1994). Para isso, Sidman (cf. 1994) tem sugerido que equivalência de estímulos pode ser um processo comportamental básico, isto é, gerado pelas contingências de reforçamento e não redutível a outros processos comportamentais.

O paradigma de equivalência tem sido usado em algumas investigações experimentais para verificar a formação de uma rede de relações numéricas equivalentes. Green (1993) ensinou dois estudantes com atraso no desenvolvimento 
cognitivo a estabelecerem diversas relações entre algarismos hindu-arábicos, quantidades de bolinhas, nome falado dos números e novos estímulos. A nomeação oral emergiu tanto para os algarismos como para as quantidades correspondentes. Green (1993) aponta que a contagem não foi um repertório necessário para a aquisição das relações numéricas.

O estudo de Green (1993) gerou uma série de investigações, todos baseados no paradigma de equivalência. Kahhale (1993), por exemplo, ensinou a pré-escolares as relações entre numerosidades de objetos e o nome falado dos números. Kahhale variou todas as possíveis dimensões secundárias dos objetos, como tamanho, forma, cor, distribuição espacial, a fim de que somente a dimensão relevante numerosidade passasse a controlar as respostas dos sujeitos. Mais tarde, Prado (1995) propôs que o paradigma de relações equivalentes poderia ser usado como instrumento na avaliação de comportamento conceitual numérico em termos de seus componentes. Prado e de Rose (1999) e Prado (2001) estenderam a proposta de 1995, verificando experimentalmente a viabilidade de uma rede de ensino de repertórios numéricos. Carmo (1997) segue a mesma linha de investigação e ensinou a pré-escolares a rede de relações numéricas, incluindo palavras escritas (nome escrito dos números) como um dos estímulos componentes do comportamento conceitual numérico adotado pela comunidade verbal. Note-se que a inclusão de palavras escritas é pertinente ao se observar que, na cultura letrada, os nomes dos números não são apenas falados, mas também escritos. Por outro lado, a definição matemática de numeral envolve qualquer símbolo usado na representação de número (algarismos hindu-arábicos, algarismos romanos, bolinhas, sinais, nomes escritos dos números, etc.).

Somente um estudo (Williams, 2000) documentado na literatura utilizou surdos como sujeitos experimentais. Williams (2000) investigou a formação de classe numérica equivalente a partir do ensino dos numerais de 1 a 6 na Linguagem Americana de Sinais (ASL), estabelecendo a relação entre estes estímulos, os numerais hindu-arábicos de 1 a 6 , nomes escritos dos números e as quantidades correspondentes. $\mathrm{O}$ trabalho pioneiro de Williams aponta para a possibilidade do ensino de comportamento conceitual numérico a indivíduos surdos a partir do acréscimo de elementos próprios da linguagem de sinais. Entretanto, esse estudo não procurou verificar a formação de classes ordinais, a partir de suas propriedades definidoras: irreflexividade, assimetria, transitividade e conectividade e nem verificou a possibilidade de substituição dos estímulos nas seqüências de numerais e símbolos da língua de sinais.

\section{Produção de seqüiências: o ensino de relações ordinais}

A definição de classes ordinais baseia-se nas propriedades das relações de ordem. Green, Stromer e Mackay (1993) propuseram um novo tipo de análise de desempenhos emergentes derivados de contingências de reforçamento simples que estabelecem a produção de sequiências de estímulos. Nesta interpretação, a análise de sequiências sob a abordagem tradicional de encadeamento, na qual um estímulo exerce uma dupla função (reforçadora para a resposta anterior e discriminativa para a resposta seguinte) como elo de ligação entre os estímulos membros de uma cadeia comportamental; ou sob a abordagem do simples controle condicional de estímulos é evitada, uma vez que não seria suficiente para uma explicação precisa acerca da produção de novas seqüências não ensinadas diretamente. A proposta alternativa desses autores enfatiza a análise das relações entre estímulos nas seqüências e entre seqüências treinadas separadamente uma da outra, e testes comportamentais que avaliam se tais relações apresentam as propriedades de uma relação ordinal (i.e: irreflexividade, assimetria, transitividade e conectividade).

Para isso, os autores definiram essas propriedades, a partir da matemática, proposta por Stevens (1951): Irreflexividade é a propriedade segundo a qual um dado elemento da sequiência não se segue a ele mesmo, devido à posição ordinal por ele ocupada. Assim, não é possível a relação A1 $\rightarrow$ A1 (em que A1 representa um determinado elemento ocupando um determinado lugar na seqüência). Assimetria que se caracteriza por uma relação unidirecional; por exemplo, se $\mathrm{A} 2 \rightarrow \mathrm{A} 3$ (lê-se $\mathrm{A} 2$ é seguido por $\mathrm{A} 3$ ), então $\mathrm{A} 3 \rightarrow \mathrm{A} 2$ (lê-se $\mathrm{A} 3$ é seguido por A2) não pode ser verdadeira para a mesma seqüência. Transitividade quando, por exemplo, A2 $\rightarrow$ A3 e A3 $\rightarrow$ A4, então A2 $\rightarrow \mathrm{A} 4$ (note-se que apenas pares de estímulos não adjacentes dentro de uma série treinada podem servir como base para inferir esta propriedade). Conectividade, que é uma relação estabelecida para todos os pares de estímulos dentro de um campo específico. Relações que exibem conexidade são necessárias (mas não são suficientes) para o arranjo de estímulos dentro de um conjunto. Por exemplo, se $\mathrm{A} 1 \rightarrow$ $\mathrm{A} 2 \rightarrow \mathrm{A} 3$, então $\mathrm{A} 1 \rightarrow \mathrm{A} 2, \mathrm{~A} 1 \rightarrow \mathrm{A} 3$, e $\mathrm{A} 2 \rightarrow \mathrm{A} 3$ (note que esta propriedade é inferida se todos os pares são possíveis, pares adjacentes e não adjacentes). Uma revisão mais exaustiva da área foi conduzida recentemente por Mackay, Stromer e Serna (1998). Nela os autores (Mackay \& cols., 1998) explicitam o termo transferência de funções enquanto uma fusão de classes arbitrárias (cf. Sidman, 1994).

Portanto, a verificação de classes ordinais pressupõe que todos os estímulos sejam mutuamente substituíveis no controle de uma mesma resposta, e que qualquer propriedade controladora exercida por um membro da classe deva ser compartilhado por todos os outros membros da mesma classe.

Por outro lado, duas perguntas têm gerado investigações experimentais: 1) existiria uma única base para o desenvolvimento de seqüências de comportamentos? 2) classes ordinais podem emergir a partir do ensino com diferentes seqüências e, portanto, intercambiar entre si seus membros fora do contexto de emparelhamento de acordo com o modelo? Em relação à primeira questão, estudos têm demonstrado que a produção de sequiências pode ser ensinada através de um treino por encadeamento de respostas. Os princípios comportamentais usados no estabelecimento de cadeias são: 1) aproximação sucessiva de cada membro, 2) colocar cada membro sob controle discriminativo, e 3) uso de estímulos com duplo papel de deixa discriminativa e reforçador condicionado, para ligar cada membro com o próximo. Uma definição mais elegante foi apresentada por Catania (1998/1999), quando afirma: "Seqüência como uma sucessão de operantes diferentes, cada uma definida pela conseqüência reforçadora de produzir uma oportunidade de emitir a próxima, até que a seqüência seja terminada por um reforçador" (p.142).

Um estudo experimental conduzido por Maydak, Stromer, Mackay e Stoddard (1995) documentou a formação de classes 
de seqüências equivalentes, o que possibilitou verificar a formação de sequiências numéricas equivalentes.

Nesse estudo (Maydak \& cols., 1995), os autores investigaram a inter-relação entre classes de estímulos e tarefas de produção de seqüência, isto é, o responder seqüencial de acordo com uma ordem pré-determinada - por exemplo: $\mathrm{a} \rightarrow \mathrm{b} \rightarrow \mathrm{c} \rightarrow \mathrm{d} ; 1 \rightarrow 2 \rightarrow 3 \rightarrow 4$, etc. Os participantes foram um homem e uma mulher de 30 e 40 anos de idade cronológica $(7$ anos 9 meses e 3 anos 6 meses, através do Peabody Picture Vocabulary Test, respectivamente). O experimento consistiu respectivamente, da formação de classes de estímulos compostos por nomes de números ditados, numerais e numerosidades (conjuntos formados por pontos). Subseqüentemente, era realizado um treino de produção de seqüêencia com as numerosidades de pontos de dois a cinco, no qual, dados conjuntos com esses números de elementos, dispostos aleatoriamente, o sujeito devia selecioná-los partindo do menos para o mais numeroso. Por meio de testes apropriados verificou-se, posteriormente, a emergência da ordenação dos numerais dois a cinco.

Em um outro estudo, Mackay, Kotlarchyk e Stromer (1997) ensinaram uma criança de 10 anos com lesão cerebral a discriminar um conjunto de dígitos e palavras correspondentes. Enquanto o professor dizia a palavra, por exemplo, "Two", o participante era ensinado a formar a palavra seqüencialmente com as letras "T", "W", "O" apresentadas na tela de um computador. Após, cada tentativa, as letras mudavam de posição na tela. Em seguida, a discriminação de outras palavras foram ensinadas gradativamente em seqüência, até completar nove palavras, todas correspondentes aos dígitos de 1 a 9 . Os autores ainda aplicaram um teste de nomeação oral (o professor apresentava um dígito ou uma palavra correspondente e perguntava: "O que é isso?"). Em seguida, o garoto era submetido a uma avaliação oral em que precisava responder seqüencialmente do menor para o maior, aos números que apareciam na tela. Embora ele tenha falado em voz alta todos os dígitos, os autores concluíram que ele não foi capaz de ordená-los apropriadamente.

Por outro lado, uma sequiência de respostas ainda poderia estar sob controle condicional. Por exemplo, na vida cotidiana poderia ser observado num boletim escolar de um estudante, $\mathrm{o}$ seguinte: Carlos tem 10 anos e está na $2^{\mathrm{a}}$ série, Antonio tem 8 anos e está na $1^{a}$ série e Francisco de 7 anos está na 3 série do ensino fundamental. Ou seja, os nomes dos alunos podem ser organizados dependendo da ordem de nascimento ou do nível de escolaridade. Assim teríamos: Francisco, Antonio e Carlos, se o critério de ordenar os nomes for pela idade do mais jovem ao mais velho, ou Antonio, Carlos e Francisco, se o critério for o nível de escolaridade da primeira para a última série.

Lazar e Kotlarchyck (1986) em estudo pioneiro demonstraram experimentalmente o controle condicional sobre o responder seqüencial, porém, adotaram como característica metodológica o ensino de apenas dois pares de estímulos (primeiros e segundos). Isso poderia levar o participante a responder por exclusão, eliminando aquele estímulo que já fizesse parte do seu repertório.

O estudo de Stromer e Mackay (1992) estendeu esses resultados, buscando estabelecer a transferência do controle condicional sem a necessidade do estabelecimento de prérequisitos para classes de equivalência via treino de empa- relhamento de acordo com o modelo. Os resultados sugerem que o treino independente de várias seqüências de respostas pode estabelecer classes de estímulos equivalentes fora do contexto de matching-to-sample.

Seguindo essa mesma linha de pesquisa, Assis e Costa (2004) também demonstraram empiricamente a emergência de relações ordinais sob controle condicional em quatro crianças normais.

Entretanto, crianças surdas têm demonstrado dificuldades no desempenho de operações aritméticas, as quais podem ser produzidas a partir de relações número-quantidade inadequadas. Adolescentes com impedimento auditivo têm demonstrado atrasos acadêmicos em relação à matemática (ver Williams, 2000). Um pré-requisito fundamental à aprendizagem de comportamentos conceituais numéricos é a ordenação. Esta habilidade pré-aritmética possibilitará o desenvolvimento de repertórios mais complexos como a contagem. Além disso, a linguagem de sinais requer não apenas discriminação sutil de movimentos, mas também utilização adequada e concomitante do espaço, o que configura em coordenação viso-espacial e um maior rigor no controle de estímulos visuais sobre o comportamento.

O presente estudo buscou avaliar a emergência do controle condicional sobre relações ordinais na presença de duas cores: verde ou vermelho, em tarefas de ordenar envolvendo a Linguagem Brasileira de Sinais (LIBRAS) para ensinar três sequiências de respostas independentes. Posteriormente, procurou-se verificar se os estímulos em cada sequiência eram funcionalmente equivalentes, ou seja, se a função exercida por um membro em uma sequiência poderia ser compartilhada por membro de uma nova sequiência na mesma posição.

\section{Método}

\section{Participantes}

Participaram do presente estudo, cinco alunos portadores de deficiência auditiva (surdez congênita), com idade variando entre 6 e 8 anos de idade, sendo três do sexo masculino e duas do sexo feminino, matriculados numa escola pública especializada. Conforme análise realizada dos dossiês de cada participante, todos apresentavam perda auditiva acima de $91 \mathrm{db}$ (surdez profunda), que foi diagnosticada através de exame audiométrico com laudo médico emitido por especialista em otorrinolaringologia. Todos os participantes eram da fase inicial de alfabetização e pertenciam à classe de baixa renda. Conforme Resolução 196/96 do Conselho Nacional de Saúde, um Termo de Consentimento Livre e Esclarecido foi assinado pelos pais dos alunos, autorizando a participação dos mesmos neste estudo.

Pré-testes, cujo objetivo era avaliar se os participantes já discriminavam cores, numerosidade (utilizados o bloco lógico marca FUNBEC com 49 peças de madeira coloridas em diferentes formas, espessuras e tamanhos), numerais em LIBRAS e nomes dos numerais em português. Durante os pré-testes, foi verificado também que os mesmos não conheciam os sinais de número em LIBRAS, numerosidades e cores verde e vermelha. Após a seleção dos participantes, a experimentadora precisou ensinar alguns sinais básicos em LIBRAS. Toda comunicação entre participantes e ex- 
perimentadora foi nesta linguagem de sinais. Abaixo segue a Tabela 1 com descrição dos participantes, por sexo, idade cronológica e a perda auditiva.

Tabela 1. Relação dos participantes por sexo, idade cronológica e perda auditiva.

\begin{tabular}{lccc}
\hline Participante & Sexo & Idade Cronológica & Perda Auditiva \\
\hline LP & Masculino & 6 anos e 6 meses & Acima de 91db \\
EG & Feminino & 6 anos e 6 meses & Acima de 91db \\
GV & Masculino & 6 anos e 6 meses & Acima de 91db \\
WS & Masculino & 6 anos e 4 meses & Acima de 91db \\
DP & Feminino & 7 anos e 7 meses & Acima de 91db \\
\hline
\end{tabular}

\section{Ambiente experimental}

Uma sala medindo aproximadamente $3,06 \mathrm{~m}$ x 3,00m x 2,90m em uma instituição pública especializada no ensino de crianças com surdez, climatizada e com relativo isolamento acústico.

\section{Material e equipamento}

No pré-teste de discriminação de numerosidade foi utilizado um conjunto de blocos lógicos de madeira, marca FUNBEC. O conjunto é constituído de 49 peças em quatro dimensões: formas (quadrado, triângulo, círculo, retângulo), cores (azul, amarelo, vermelho), tamanhos (grande, pequeno) e espessuras (grosso, fino). Após dispor as peças sobre uma mesa, a experimentadora solicitava ao aluno que apontasse qual o conjunto apresentava mais peças com formas, cores, tamanhos ou espessuras diferentes. Cada conjunto de peças com diferentes dimensões era apresentado três vezes consecutivas, aleatoriamente (ver Tabela 2).

Tabela 2. Número de respostas corretas por participante, pelo número de tentativas em cada conjunto de peças nas quatro dimensões.

\begin{tabular}{lcccc}
\hline \multirow{2}{*}{ Participante } & \multicolumn{4}{c}{ Conjunto de Peças / Dimensões } \\
\cline { 2 - 5 } & Formas & Cores & Tamanho & Espessura \\
\hline LP & $1 / 3$ & $0 / 3$ & $0 / 3$ & $1 / 3$ \\
EG & $0 / 3$ & $0 / 3$ & $1 / 3$ & $0 / 3$ \\
GV & $0 / 3$ & $1 / 3$ & $1 / 3$ & $0 / 3$ \\
WS & $0 / 3$ & $0 / 3$ & $0 / 3$ & $0 / 3$ \\
DP & $1 / 3$ & $0 / 3$ & $0 / 3$ & $0 / 3$ \\
\hline
\end{tabular}

Um outro pré-teste utilizou-se cartões com sinais em LIBRAS e o conjunto de peças da FUNBEC. Por exemplo, na presença do sinal "um" em LIBRAS apresentado pela experimentadora, o participante apontava dentre os três conjuntos de peças, qual o conjunto que correspondia à quantidade indicada através do sinal em LIBRAS. Cada tentativa envolvia apenas um sinal em LIBRAS, que era apresentado três vezes consecutivas (ver Tabela 3 ).
Tabela 3. Número de respostas corretas por participante, pelo número de tentativas em cada conjunto de sinais em LIBRAS.

\begin{tabular}{|c|c|c|c|c|c|c|}
\hline \multirow{2}{*}{ Participante } & \multicolumn{6}{|c|}{ Sinais em LIBRAS } \\
\hline & $c^{\lambda}$ & $a$ & $\approx$ & $\mathrm{E}$ & श & / \\
\hline LP & $1 / 3$ & $0 / 3$ & $0 / 3$ & $1 / 3$ & $0 / 3$ & $0 / 3$ \\
\hline EG & $0 / 3$ & $0 / 3$ & $1 / 3$ & $0 / 3$ & $0 / 3$ & $0 / 3$ \\
\hline $\mathrm{GV}$ & $0 / 3$ & $1 / 3$ & $1 / 3$ & $0 / 3$ & $0 / 3$ & $0 / 3$ \\
\hline WS & $0 / 3$ & $0 / 3$ & $0 / 3$ & $0 / 3$ & $0 / 3$ & $0 / 3$ \\
\hline DP & $1 / 3$ & $0 / 3$ & $0 / 3$ & $0 / 3$ & $0 / 3$ & $0 / 3$ \\
\hline
\end{tabular}

Nas etapas de treino e testes, o controle da apresentação dos estímulos, número de tentativas, número de posições de cada estímulo na tela, registro de respostas corretas e incorretas foi utilizado um software (REL 3.0 for windows) construído para esta pesquisa. Um microcomputador notebook Toshiba, MMX com 32 MB de memória RAM, tela de matriz dual scan de 12 " com mouse utilizado para coleta dos dados comportamentais.

\section{Estímulos}

Além dos blocos lógicos e cartões empregados no pré-teste, foram utilizados três conjuntos de estímulos. O conjunto "A", formado pelo nome escrito dos números (A1, A2, A3, A4, A5, A6). O conjunto "B" (B1, B2, B3, B4, B5, B6) formado por numerais representados em LIBRAS (sinais), e o conjunto "C" $(\mathrm{C} 1, \mathrm{C} 2, \mathrm{C} 3, \mathrm{C} 4, \mathrm{C} 5, \mathrm{C} 6)$, com formas abstratas (figuras não usuais). A seguir, a Figura 1 apresenta os estímulos que foram usados no estudo com a adição dos estímulos condicionais nas cores verde ou vermelha, que ficava numa "janela central" medindo aproximadamente 2,5 cm x 2,5 cm, acima da "área de construção".

\begin{tabular}{|c|c|c|c|c|c|}
\hline $\begin{array}{l}\text { A1 } \\
\text { Um }\end{array}$ & $\begin{array}{c}\text { A2 } \\
\text { Dois }\end{array}$ & $\begin{array}{c}\mathbf{A 3} \\
\text { Três }\end{array}$ & $\begin{array}{c}\text { A4 } \\
\text { Quatro }\end{array}$ & $\begin{array}{c}\text { A5 } \\
\text { Cinco }\end{array}$ & $\begin{array}{c}\text { A6 } \\
\text { Seis }\end{array}$ \\
\hline B1 & B2 & B3 & B4 & B5 & B6 \\
\hline$c^{\lambda}$ & $-\pi$ & $\approx$ & 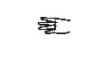 & श) & 但 \\
\hline C1 & C2 & C3 & C4 & C5 & C6 \\
\hline $\mathbf{W}$ & Uu & U U & $\begin{array}{l}\mathbf{U} \\
\mathbf{U}\end{array}$ & ${ }_{\mathbf{w}}^{\mathbf{w}_{u}} \mathbf{u}$ & $\begin{array}{l}\text { UUL } \\
\text { UUU }\end{array}$ \\
\hline
\end{tabular}

Figura 1. Conjunto de estímulos utilizados no estudo

Para a apresentação dos estímulos, houve a divisão da tela do computador em duas partes assim definidas: "área de escolha" (parte inferior da tela), composta de oito quadrados, sendo quatro em cima e quatro embaixo, cada um medindo aproximadamente 4,5 cm x 4,5 cm, onde os estímulos eram apresentados de forma aleatória, e "área de construção" (parte superior da tela do computador), consistia na formação completa da seqüência após o "tocar" no estímulo que se encontrava na "área de escolha", que também era composta de oito quadrados medindo aproximadamente 2,5 cm x 2,5 cm cada. E acima da "área de construção" tinha uma janela medindo aproximadamente $2,5 \mathrm{~cm}$ x 2,5 cm, com a cor "verde ou vermelha" dentro dela. 


\section{Procedimento}

A Tabela 4 apresenta um sumário do delineamento experimental com os procedimentos de treino por encadea- mento, tipos de tentativas, critério de acerto, probabilidade de reforços, testes de seqüenciação, testes de transitividade e de conectividade na presença das cores verde ou vermelha com os estímulos do conjunto "A" e "B" e "C".

Tabela 4. Sumário dos procedimentos de treino e testes com o número de fases, tipos de tentativas, tipo de estímulo, critério de acerto e probabilidade de reforços.

\begin{tabular}{|c|c|c|c|c|c|}
\hline Fase & Tipo de Bloco & Tipo de tentativas em seqüiência & $\begin{array}{l}\text { Estímulo } \\
\text { Condicional } \\
\text { "Cores" }\end{array}$ & $\begin{array}{l}\text { Critério de } \\
\text { Acerto }\end{array}$ & $\begin{array}{c}\text { Probabilidade de } \\
\text { Reforços }\end{array}$ \\
\hline 1 & Treino de Encadeamento do conjunto A & $\mathrm{A} 1 \rightarrow \mathrm{A} 2 \rightarrow \mathrm{A} 3 \rightarrow \mathrm{A} 4 \rightarrow \mathrm{A} 5 \rightarrow \mathrm{A} 6$ & Verde & $3 / 3$ & $1 / 1$ \\
\hline 2 & Teste de Seqüenciação do conjunto A & $\mathrm{A} 1 \rightarrow \mathrm{A} 2 \rightarrow \mathrm{A} 3 \rightarrow \mathrm{A} 4 \rightarrow \mathrm{A} 5 \rightarrow \mathrm{A} 6$ & Verde & - & - \\
\hline 3 & Treino de Encadeamento do conjunto A & $\mathrm{A} 6 \rightarrow \mathrm{A} 5 \rightarrow \mathrm{A} 4 \rightarrow \mathrm{A} 3 \rightarrow \mathrm{A} 2 \rightarrow \mathrm{A} 1$ & Vermelho & $3 / 3$ & $1 / 1$ \\
\hline 4 & Teste de Seqüenciação & $\mathrm{A} 6 \rightarrow \mathrm{A} 5 \rightarrow \mathrm{A} 4 \rightarrow \mathrm{A} 3 \rightarrow \mathrm{A} 2 \rightarrow \mathrm{A} 1$ & Vermelho & - & - \\
\hline 5 & Treino de Encadeamento do conjunto B & $\mathrm{B} 1 \rightarrow \mathrm{B} 2 \rightarrow \mathrm{B} 3 \rightarrow \mathrm{B} 4 \rightarrow \mathrm{B} 5 \rightarrow \mathrm{B} 6$ & Verde & $3 / 3$ & $1 / 1$ \\
\hline 6 & Teste de Seqüenciação & $\mathrm{B} 1 \rightarrow \mathrm{B} 2 \rightarrow \mathrm{B} 3 \rightarrow \mathrm{B} 4 \rightarrow \mathrm{B} 5 \rightarrow \mathrm{B} 6$ & Verde & & - \\
\hline 7 & Treino de Encadeamento do conjunto B & $\mathrm{B} 6 \rightarrow \mathrm{B} 5 \rightarrow \mathrm{B} 4 \rightarrow \mathrm{B} 3 \rightarrow \mathrm{B} 2 \rightarrow \mathrm{B} 1$ & Vermelho & $3 / 3$ & $1 / 1$ \\
\hline 8 & Teste de Seqüenciação & $\mathrm{B} 6 \rightarrow \mathrm{B} 5 \rightarrow \mathrm{B} 4 \rightarrow \mathrm{B} 3 \rightarrow \mathrm{B} 2 \rightarrow \mathrm{B} 1$ & Vermelho & - & - \\
\hline 9 & Treino de Encadeamento do conjunto C & $\mathrm{C} 1 \rightarrow \mathrm{C} 2 \rightarrow \mathrm{C} 3 \rightarrow \mathrm{C} 4 \rightarrow \mathrm{C} 5 \rightarrow \mathrm{C} 6$ & Verde & $3 / 3$ & $1 / 1$ \\
\hline 10 & Teste de Seqüenciação & $\mathrm{C} 1 \rightarrow \mathrm{C} 2 \rightarrow \mathrm{C} 3 \rightarrow \mathrm{C} 4 \rightarrow \mathrm{C} 5 \rightarrow \mathrm{C} 6$ & Verde & - & - \\
\hline 11 & Treino de Encadeamento do conjunto C & $\mathrm{C} 6 \rightarrow \mathrm{C} 5 \rightarrow \mathrm{C} 4 \rightarrow \mathrm{C} 3 \rightarrow \mathrm{C} 2 \rightarrow \mathrm{C} 1$ & Vermelho & $3 / 3$ & $1 / 1$ \\
\hline 12 & Teste de Seqüenciação & $\mathrm{C} 6 \rightarrow \mathrm{C} 5 \rightarrow \mathrm{C} 4 \rightarrow \mathrm{C} 3 \rightarrow \mathrm{C} 2 \rightarrow \mathrm{C} 1$ & Vermelho & - & - \\
\hline \multirow[t]{2}{*}{13} & \multirow[t]{2}{*}{ Teste de transitividade do conjunto A } & $\begin{array}{l}\mathrm{A} 1 \rightarrow \mathrm{A} 3 \\
\mathrm{~A} 1 \rightarrow \mathrm{A} 4 \\
\mathrm{~A} 1 \rightarrow \mathrm{A} 5 \\
\mathrm{~A} 1 \rightarrow \mathrm{A} 6 \\
\mathrm{~A} 2 \rightarrow \mathrm{A} 4 \\
\mathrm{~A} 2 \rightarrow \mathrm{A} 5 \\
\mathrm{~A} 2 \rightarrow \mathrm{A} 6 \\
\mathrm{~A} 3 \rightarrow \mathrm{A} 5 \\
\mathrm{~A} 3 \rightarrow \mathrm{A} 6 \\
\mathrm{~A} 4 \rightarrow \mathrm{A} 6\end{array}$ & Verde & - & - \\
\hline & & $\begin{array}{l}\mathrm{A} 6 \rightarrow \mathrm{A} 4 \\
\mathrm{~A} 6 \rightarrow \mathrm{A} 3 \\
\mathrm{~A} 6 \rightarrow \mathrm{A} 2 \\
\mathrm{~A} 6 \rightarrow \mathrm{A} 1 \\
\mathrm{~A} 5 \rightarrow \mathrm{A} 3 \\
\mathrm{~A} 5 \rightarrow \mathrm{A} 2 \\
\mathrm{~A} 5 \rightarrow \mathrm{A} 1 \\
\mathrm{~A} 4 \rightarrow \mathrm{A} 2 \\
\mathrm{~A} 4 \rightarrow \mathrm{A} 1 \\
\mathrm{~A} 3 \rightarrow \mathrm{A} 1\end{array}$ & Vermelho & - & - \\
\hline \multirow[t]{2}{*}{14} & \multirow[t]{2}{*}{ Teste de transitividade do conjunto $\mathrm{B}$} & $\begin{array}{l}\mathrm{B} 1 \rightarrow \mathrm{B} 3 \\
\mathrm{~B} 1 \rightarrow \mathrm{B} 4 \\
\mathrm{~B} 1 \rightarrow \mathrm{B} 5 \\
\mathrm{~B} 1 \rightarrow \mathrm{B} 6 \\
\mathrm{~B} 2 \rightarrow \mathrm{B} 4 \\
\mathrm{~B} 2 \rightarrow \mathrm{B} 5 \\
\mathrm{~B} 2 \rightarrow \mathrm{B} 6 \\
\mathrm{~B} 3 \rightarrow \mathrm{B} 5 \\
\mathrm{~B} 3 \rightarrow \mathrm{B} 6 \\
\mathrm{~B} 4 \rightarrow \mathrm{B} 6\end{array}$ & Verde & - & - \\
\hline & & $\begin{array}{l}\mathrm{B} 6 \rightarrow \mathrm{B} 4 \\
\mathrm{~B} 6 \rightarrow \mathrm{B} 3 \\
\mathrm{~B} 6 \rightarrow \mathrm{B} 2 \\
\mathrm{~B} 6 \rightarrow \mathrm{B} 1 \\
\mathrm{~B} 5 \rightarrow \mathrm{B} 3 \\
\mathrm{~B} 5 \rightarrow \mathrm{B} 2 \\
\mathrm{~B} 5 \rightarrow \mathrm{B} 1 \\
\mathrm{~B} 4 \rightarrow \mathrm{B} 2 \\
\mathrm{~B} 4 \rightarrow \mathrm{B} 1 \\
\mathrm{~B} 3 \rightarrow \mathrm{B} 1\end{array}$ & vermelho & - & - \\
\hline
\end{tabular}




\begin{tabular}{|c|c|c|c|c|c|}
\hline Fase & Tipo de Bloco & Tipo de tentativas em seqüência & $\begin{array}{l}\text { Estímulo } \\
\text { Condicional } \\
\text { "Cores" }\end{array}$ & $\begin{array}{l}\text { Critério de } \\
\text { Acerto }\end{array}$ & $\begin{array}{l}\text { Probabilidade de } \\
\text { Reforços }\end{array}$ \\
\hline 15 & Teste de transitividade do conjunto $\mathrm{C}$ & $\begin{array}{l}\mathrm{C} 1 \rightarrow \mathrm{C} 3 \\
\mathrm{C} 1 \rightarrow \mathrm{C} 4 \\
\mathrm{C} 1 \rightarrow \mathrm{C} 5 \\
\mathrm{C} 1 \rightarrow \mathrm{C} 6 \\
\mathrm{C} 2 \rightarrow \mathrm{C} 4 \\
\mathrm{C} 2 \rightarrow \mathrm{C} 5 \\
\mathrm{C} 2 \rightarrow \mathrm{C} 6 \\
\mathrm{C} 3 \rightarrow \mathrm{C} 5 \\
\mathrm{C} 3 \rightarrow \mathrm{C} 6 \\
\mathrm{C} 4 \rightarrow \mathrm{C} 6 \\
\mathrm{C} 6 \rightarrow \mathrm{C} 4 \\
\mathrm{C} 6 \rightarrow \mathrm{C} 3 \\
\mathrm{C} 6 \rightarrow \mathrm{C} 2 \\
\mathrm{C} 6 \rightarrow \mathrm{C} 1 \\
\mathrm{C} 5 \rightarrow \mathrm{C} 3 \\
\mathrm{C} 5 \rightarrow \mathrm{C} 2 \\
\mathrm{C} 5 \rightarrow \mathrm{C} 1 \\
\mathrm{C} 4 \rightarrow \mathrm{C} 2 \\
\mathrm{C} 4 \rightarrow \mathrm{C} 1 \\
\mathrm{C} 3 \rightarrow \mathrm{C} 1\end{array}$ & 更 & - & - \\
\hline 16 & $\begin{array}{l}\text { Revisão de linha de base dos estímulos dos } \\
\text { conjuntos A e B }\end{array}$ & $\begin{array}{l}\mathrm{A} 1 \rightarrow \mathrm{A} 2 \rightarrow \mathrm{A} 3 \rightarrow \mathrm{A} 4 \rightarrow \mathrm{A} 5 \rightarrow \mathrm{A} 6 \\
\mathrm{~A} 6 \rightarrow \mathrm{A} 5 \rightarrow \mathrm{A} 4 \rightarrow \mathrm{A} 3 \rightarrow \mathrm{A} 2 \rightarrow \mathrm{A} 1 \\
\mathrm{~B} 1 \rightarrow \mathrm{B} 2 \rightarrow \mathrm{B} 3 \rightarrow \mathrm{B} 4 \rightarrow \mathrm{B} 5 \rightarrow \mathrm{B} 6 \\
\mathrm{~B} 6 \rightarrow \mathrm{B} 5 \rightarrow \mathrm{B} 4 \rightarrow \mathrm{B} 3 \rightarrow \mathrm{B} 2 \rightarrow \mathrm{B} 1\end{array}$ & $\begin{array}{l}\text { Verde } \\
\text { Vermelho } \\
\text { Verde } \\
\text { Vermelho }\end{array}$ & $3 / 3$ & $1 / 1$ \\
\hline 17 & Teste de conectividade $\mathrm{A} / \mathrm{B}$ e $\mathrm{B} / \mathrm{A}$ & $\begin{array}{l}\mathrm{A} 1 \rightarrow \mathrm{B} 2 \rightarrow \mathrm{A} 3 \rightarrow \mathrm{B} 4 \rightarrow \mathrm{A} 5 \rightarrow \mathrm{B} 6 \\
\mathrm{~B} 6 \rightarrow \mathrm{A} 5 \rightarrow \mathrm{B} 4 \rightarrow \mathrm{A} 3 \rightarrow \mathrm{B} 2 \rightarrow \mathrm{A} 1 \\
\mathrm{~B} 1 \rightarrow \mathrm{A} 2 \rightarrow \mathrm{B} 3 \rightarrow \mathrm{A} 4 \rightarrow \mathrm{B} 5 \rightarrow \mathrm{A} 6 \\
\mathrm{~A} 6 \rightarrow \mathrm{B} 5 \rightarrow \mathrm{A} 4 \rightarrow \mathrm{B} 3 \rightarrow \mathrm{A} 2 \rightarrow \mathrm{B} 1\end{array}$ & $\begin{array}{l}\text { Verde } \\
\text { Vermelho } \\
\text { Verde } \\
\text { Vermelho }\end{array}$ & - & - \\
\hline 18 & Revisão de linha de base de A e C & $\begin{array}{l}\mathrm{A} 1 \rightarrow \mathrm{A} 2 \rightarrow \mathrm{A} 3 \rightarrow \mathrm{A} 4 \rightarrow \mathrm{A} 5 \rightarrow \mathrm{A} 6 \\
\mathrm{~A} 6 \rightarrow \mathrm{A} 5 \rightarrow \mathrm{A} 4 \rightarrow \mathrm{A} 3 \rightarrow \mathrm{A} 2 \rightarrow \mathrm{A} 1 \\
\mathrm{C} 1 \rightarrow \mathrm{C} 2 \rightarrow \mathrm{C} 3 \rightarrow \mathrm{C} 4 \rightarrow \mathrm{C} 5 \rightarrow \mathrm{C} 6 \\
\mathrm{C} 6 \rightarrow \mathrm{C} 5 \rightarrow \mathrm{C} 4 \rightarrow \mathrm{C} 3 \rightarrow \mathrm{C} 2 \rightarrow \mathrm{C} 1\end{array}$ & $\begin{array}{l}\text { Verde } \\
\text { Vermelho } \\
\text { Verde } \\
\text { Vermelho }\end{array}$ & $3 / 3$ & $1 / 1$ \\
\hline 19 & Teste de conectividade $\mathrm{A} / \mathrm{C} \mathrm{C/A}$ & $\begin{array}{l}\mathrm{A} 1 \rightarrow \mathrm{C} 2 \rightarrow \mathrm{A} 3 \rightarrow \mathrm{C} 4 \rightarrow \mathrm{A} 5 \rightarrow \mathrm{C} 6 \\
\mathrm{C} 6 \rightarrow \mathrm{A} 5 \rightarrow \mathrm{C} 4 \rightarrow \mathrm{A} 3 \rightarrow \mathrm{C} 2 \rightarrow \mathrm{A} 1 \\
\mathrm{C} 1 \rightarrow \mathrm{A} 2 \rightarrow \mathrm{C} 3 \rightarrow \mathrm{A} 4 \rightarrow \mathrm{C} 5 \rightarrow \mathrm{A} 6 \\
\mathrm{~A} 6 \rightarrow \mathrm{C} 5 \rightarrow \mathrm{A} 4 \rightarrow \mathrm{C} 3 \rightarrow \mathrm{A} 2 \rightarrow \mathrm{C} 1\end{array}$ & $\begin{array}{l}\text { Verde } \\
\text { Vermelho } \\
\text { Verde } \\
\text { Vermelho }\end{array}$ & - & - \\
\hline 20 & Revisão de linha de base de "B" e "C" & $\begin{array}{l}\mathrm{B} 1 \rightarrow \mathrm{B} 2 \rightarrow \mathrm{B} 3 \rightarrow \mathrm{B} 4 \rightarrow \mathrm{B} 5 \rightarrow \mathrm{B} 6 \\
\mathrm{~B} 6 \rightarrow \mathrm{B} 5 \rightarrow \mathrm{B} 4 \rightarrow \mathrm{B} 3 \rightarrow \mathrm{B} 2 \rightarrow \mathrm{B} 1 \\
\mathrm{C} 1 \rightarrow \mathrm{C} 2 \rightarrow \mathrm{C} 3 \rightarrow \mathrm{C} 4 \rightarrow \mathrm{C} 5 \rightarrow \mathrm{C} 6 \\
\mathrm{C} 6 \rightarrow \mathrm{C} 5 \rightarrow \mathrm{C} 4 \rightarrow \mathrm{C} 3 \rightarrow \mathrm{C} 2 \rightarrow \mathrm{C} 1\end{array}$ & $\begin{array}{l}\text { Verde } \\
\text { Vermelho } \\
\text { Verde } \\
\text { Vermelho }\end{array}$ & $3 / 3$ & $1 / 1$ \\
\hline 21 & Teste de conectividade $\mathrm{B} / \mathrm{C} \mathrm{C} / \mathrm{B}$ & $\begin{array}{l}\mathrm{B} 1 \rightarrow \mathrm{C} 2 \rightarrow \mathrm{B} 3 \rightarrow \mathrm{C} 4 \rightarrow \mathrm{B} 5 \rightarrow \mathrm{C} 6 \\
\mathrm{C} 6 \rightarrow \mathrm{B} 5 \rightarrow \mathrm{C} 4 \rightarrow \mathrm{B} 3 \rightarrow \mathrm{C} 2 \rightarrow \mathrm{B} 1 \\
\mathrm{C} 1 \rightarrow \mathrm{B} 2 \rightarrow \mathrm{C} 3 \rightarrow \mathrm{B} 4 \rightarrow \mathrm{C} 5 \rightarrow \mathrm{B} 6 \\
\mathrm{~B} 6 \rightarrow \mathrm{C} 5 \rightarrow \mathrm{B} 4 \rightarrow \mathrm{C} 3 \rightarrow \mathrm{B} 2 \rightarrow \mathrm{C} 1\end{array}$ & $\begin{array}{l}\text { Verde } \\
\text { Vermelho } \\
\text { Verde } \\
\text { Vermelho }\end{array}$ & - & - \\
\hline
\end{tabular}

Fase 1: Treino de Encadeamento de respostas com o conjunto de estímulos "A", na presença da cor verde, que consistia em o participante tocar numa ordem crescente, cada figura na seqüência programada (por exemplo: A $1 \rightarrow$ A2). Após responder corretamente esta sequiência, três vezes consecutivas, sem erro, um novo estímulo era introduzido (por exemplo: $\mathrm{A} 1 \rightarrow \mathrm{A} 2 \rightarrow \mathrm{A} 3$ ) e assim por diante, até ordenar todos os seis estímulos. Somente ao responder a última figura disponível em cada seqüência programada, o reforço social era liberado. O participante e a experimentadora sentavam-se em frente ao microcomputador, a experimentadora permanecia um pouco mais à frente do participante para que o mesmo pudesse ter boa visualização dos sinais emitidos. A experimentadora fornecia a seguinte instrução mínima ao participante (através da LIBRAS): "Olhar", "Prestar atenção", e "Tocar na cor". Todos os estímulos apareciam 
na "área de escolha". O critério de acertos era de três vezes consecutivas, sem erros, após cada sequiência programada (dois, três, quatro cinco ou seis estímulos). Os estímulos eram introduzidos gradativamente. A experimentadora solicitava ao participante para que tocasse na figura (esta se deslocava para o primeiro quadrado da "área de construção"). Caso a resposta fosse correta, havia uma conseqüência (uma animação gráfica na tela) durante 5 s e paralelamente a experimentadora fornecia consequiências verbais em LIBRAS com as frases: "Muito Bem", "Certo" e "Legal". Essas conseqüências eram fornecidas após cada seqüência correta. Qualquer outra resposta emitida pelo participante na tela embranquecia por $1 \mathrm{~s}$ (intervalo entre tentativas) e uma nova configuração de estímulos (entende-se por configuração de estímulos a apresentação aleatória dos estímulos no painel de escolha, após cada tentativa) era apresentada na "área de escolha". O número de tentativas (uma tentativa consistia em tocar todos os estímulos apresentados na "área de escolha") em cada seqüência programada era de 10, no máximo.

Fase 2: Teste de Seqüenciação do conjunto "A", na presença da cor verde. A experimentadora solicitava que o participante tocasse na cor verde. Em seguida as figuras eram apresentadas simultaneamente na "área de escolha" que seriam deslocadas para a "área de construção" à medida que o participante tocasse uma a uma. O participante era informando através de sinais em LIBRAS que agora não havia "animação gráfica"; ou seja, não havia conseqüenciação após responder toda seqüência programada; caso a resposta fosse correta, esta era registrada prontamente e o participante era exposto à fase seguinte. Caso a resposta não fosse correta, uma nova configuração dos estímulos era reapresentada. Se o participante respondesse corretamente nesta segunda tentativa, registrava-se como uma resposta com atraso. Este tinha como objetivo verificar se o participante tinha aprendido a tocar numa ordem crescente um conjunto de estímulos, sem conseqüência reforçadora na presença da cor verde.

Fase 3: Treino de Encadeamento do conjunto "A" na presença da cor vermelha. Os mesmos parâmetros utilizados na Fase 1 foram os da presente fase, à exceção do estímulo condicional que passou a ser na cor vermelha, que consistia no participante tocar a figura que estava na "área de escolha", numa ordem decrescente, até que a seqüência de respostas fosse concluída.

Fase 4: Teste de Seqüenciação do conjunto "A", na presença da cor vermelha. Os mesmos parâmetros utilizados na Fase 2 foram os da presente fase, com exceção do estímulo condicional que passou a ser na cor vermelha, que consistia no participante tocar a figura que estava na "área de escolha", numa ordem decrescente, até que a seqüência de respostas fosse concluída.

Fase 5: Treino de Encadeamento do conjunto "B", na presença da cor verde. Os mesmos parâmetros utilizados na Fase 1 eram os da presente fase, à exceção dos estímulos que passaram a ser os sinais em LIBRAS correspondentes aos numerais.

Fase 6: Teste de Seqüenciação do conjunto "B", na presença da cor verde. Os mesmos parâmetros utilizados na Fase 2 eram os da presente fase, à exceção dos estímulos que passaram a ser os sinais em LIBRAS correspondentes aos numerais.
Fase 7: Treino de Encadeamento do conjunto "B", na presença da cor vermelha. Os mesmos parâmetros utilizados na Fase 3 eram os da presente fase, com exceção dos estímulos do conjunto "B" e do estímulo condicional que passou a ser na cor vermelha, que consistia no participante tocar a figura que estava na "área de escolha", numa ordem decrescente, até que uma seqüência de respostas fosse concluída.

Fase 8: Teste de Seqüenciação do conjunto B, na presença da cor vermelha. Os mesmos parâmetros utilizados na Fase 2 eram os da presente fase, com exceção dos estímulos do conjunto "B" e do estímulo condicional que passou a ser na cor vermelha, que consistia no participante tocar a figura que estava na "área de escolha", numa ordem decrescente, até que a sequiência de respostas fosse concluída.

Fase 9: Treino de Encadeamento do conjunto "C", na presença da cor verde. Os mesmos parâmetros utilizados nas fases anteriores eram os da presente fase, à exceção dos estímulos que passaram a ser com formas abstratas (numerosidade).

Fase 10: Teste de Seqüênciação, na presença da cor verde. Os mesmos parâmetros utilizados nas fases anteriores eram os da presente fase, à exceção dos estímulos que passaram a ser com formas abstratas (numerosidade).

Fase 11: Treino de Encadeamento do conjunto "C", na presença da cor vermelha. Os mesmos parâmetros utilizados nas fases anteriores eram os da presente fase, à exceção dos estímulos que passaram a ser com formas abstratas (numerosidade).

Fase 12: Teste de Seqüênciação, na presença da cor vermelha. Os mesmos parâmetros utilizados nas fases anteriores eram os da presente fase, à exceção dos estímulos que passaram a ser com formas abstratas (numerosidade).

Fase 13: Testes de transitividade envolvendo os estímulos do conjunto "A", na presença da cor verde. A experimentadora solicitava ao participante que tocasse na cor verde, em seguida apareciam na "área de escolha" os pares de estímulos a cada tentativa, numa ordem crescente, por exemplo, $\mathrm{A} 1 \rightarrow \mathrm{A} 3$. Caso o participante não conseguisse responder com acerto, a tela embranquecia e uma nova configuração aparecia na tela. Não houve consequiência diferencial. Testes de transitividade com os estímulos do conjunto "A", na presença da cor vermelha. Nesta fase, foram seguidas as mesmas orientações da fase anterior, com a diferença no tipo de estímulo condicional na cor vermelha, que consistia em o participante tocar na presença da cor vermelha, cada estímulo numa ordem decrescente, por exemplo, A3 $\rightarrow$ A1 até que toda a seqüência estivesse concluída.

Fase 14: Testes de transitividade envolvendo os estímulos do conjunto "B", na presença da cor verde. Nesta fase, foram seguidas as mesmas orientações da fase 13 , com a diferença no tipo de estímulo do conjunto "B". Testes de transitividade com estímulos do conjunto "B", na presença da cor vermelha. Nesta fase, foram seguidas as mesmas orientações da fase 13, com a diferença no tipo de estímulo do conjunto "B".

Fase 15: Testes de transitividade com estímulos do conjunto "C", na presença da cor verde. Nesta fase, foram seguidas as mesmas orientações da fase 13, com a diferença no tipo de estímulo do conjunto " $C$ ". Testes de transitividade com estímulos do conjunto "C", na presença da cor vermelha. Nesta fase, foram seguidas as mesmas orientações da fase 13, com a diferença no tipo de estímulo do conjunto " $\mathrm{C}$ ". 
Fase 16: Revisão de linha de base dos estímulos dos conjuntos "A" na presença da cor verde. Esta fase consistia em o participante tocar na cor verde, os estímulos eram apresentados na "área de escolha" numa ordem crescente e deveriam ser selecionados pelo participante. Caso o participante respondesse correto ou incorretamente, os mesmos critérios da fase de treino anterior foram adotados.

A revisão de linha de base com os estímulos do conjunto "B" com a cor verde seguiu os mesmos critérios da revisão anterior.

Na revisão de linha de base com estímulos do conjunto "A" na presença da cor vermelha, também seguiu os mesmos critérios da revisão anterior. Agora o participante deveria tocar os estímulos numa ordem inversa.

Revisão de linha de base com estímulos do conjunto "B" na presença da cor vermelha. Utilizou-se os mesmos critérios da revisão anterior. O participante deveria responder numa ordem inversa.

Fase 17: Teste de conectividade na relação de A/B na presença da cor verde. Teste que consistia na presença da cor verde com os estímulos dos dois conjuntos (A e B) na "área de escolha", randomizados, numa ordem crescente, por exemplo, $\mathrm{A} 1 \rightarrow \mathrm{B} 2 \rightarrow \mathrm{A} 3 \rightarrow \mathrm{B} 4 \rightarrow \mathrm{A} 5 \rightarrow \mathrm{B} 6$. O participante deveria tocar um a um gradativamente, até que fosse completada a sequiência na "área de construção". Caso a resposta fosse incorreta, a tela embranquecia e haveria mais uma re-exposição. Neste teste, o objetivo era verificar se novas relações emergiam sem qualquer treino adicional e se os estímulos seriam intercambiáveis funcionalmente (ver na Figura 2 um exemplo de tentativa). Teste de conectividade na relação de $\mathrm{B} / \mathrm{A}$ na presença da cor verde. Nesta fase, foram seguidas as mesmas orientações do teste anterior.

Teste de conectividade na relação de $\mathrm{A} / \mathrm{B}$, na presença da cor vermelha. Nesta fase, foram seguidas as mesmas orientações do teste anterior, com a diferença no tipo de estímulo condicional na cor vermelha, o participante deveria tocar os estímulos numa ordem decrescente, por exemplo, A6 $\rightarrow \mathrm{B} 5 \rightarrow$ $\mathrm{A} 4 \rightarrow \mathrm{B} 3 \rightarrow \mathrm{A} 2 \rightarrow \mathrm{B} 1$. Teste de conectividade na relação de B/ $A$, na presença da cor vermelha. Nesta fase, foram seguidas as mesmas orientações do teste anterior (ver na Figura 3 um exemplo de tentativa).

Fase 18: Revisão de linha de base de A e C na presença da cor verde. Foram usados os mesmos parâmetros dos treinos anteriores. Na revisão de linha de base de A e C na presença da cor vermelha, foram usados os mesmos parâmetros dos treinos anteriores.

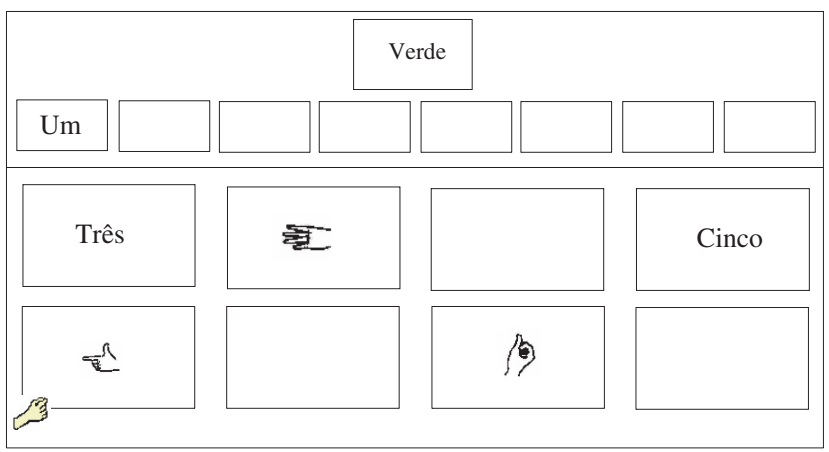

Figura 2. Exemplo de uma tentativa de teste de conectividade A/B na presença da cor verde.

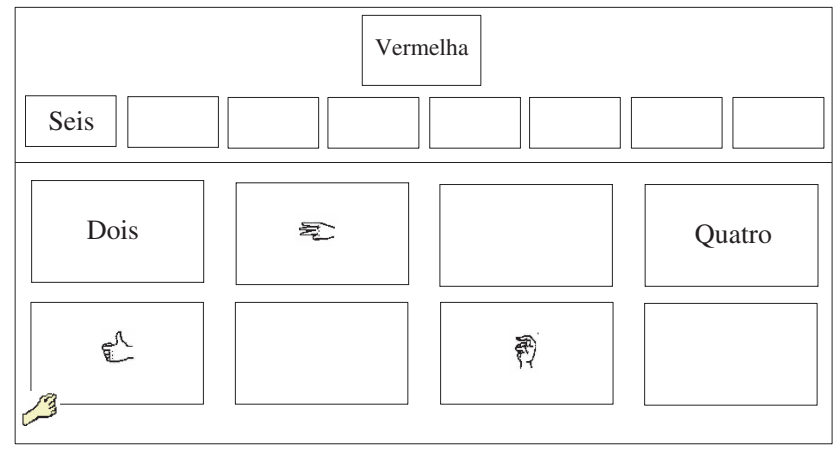

Figura 3. Exemplo de uma tentativa de teste de conectividade A/B na presença da cor vermelha.

Fase 19: Teste de conectividade A/C e C/A na presença da cor verde. Foram usados os mesmos parâmetros dos testes anteriores. Nos teste de conectividade $\mathrm{A} / \mathrm{C}$ e $\mathrm{C} / \mathrm{A}$ na presença da cor vermelha foram usados os mesmos parâmetros dos testes anteriores.

Fase 20: Revisão de linha de base das seqüências "B" e " $\mathrm{C}$ na presença da cor verde. Eram usados os mesmos parâmetros dos treinos anteriores. Em seguida era conduzida a revisão de linha de base das sequiências "B" e "C" na presença da cor vermelha. Eram usados os mesmos parâmetros dos treinos anteriores.

Fase 21: Teste de conectividade $\mathrm{B} / \mathrm{C}$ e $\mathrm{C} / \mathrm{B}$ na presença da cor verde. Foram usados os mesmos parâmetros dos testes anteriores. Em seguida, teste de conectividade B/C e C/B na presença da cor vermelha eram aplicados. Eram usados os mesmos parâmetros dos testes anteriores.

\section{Resultados}

A Tabela 5 apresenta o resultado dos cinco participantes durante o treino por encadeamento na última seqüência ensinada, na presença das cores verde ou vermelho. Dois participantes (EG e LP) alcançaram o critério de acerto adotado pela experimentadora em todas as seqüências previstas. Os demais precisaram de mais exposições ao treino para alcançar o critério de acerto. O participante DP precisou de sete exposições à seqüência $\mathrm{B} 1 \rightarrow \mathrm{B} 2 \rightarrow \mathrm{B} 3 \rightarrow \mathrm{B} 4 \rightarrow \mathrm{B} 5 \rightarrow$ $\mathrm{B} 6$ na presença da cor verde e oito vezes na sequiência $\mathrm{B} 6 \rightarrow$ $\mathrm{B} 5 \rightarrow \mathrm{B} 4 \rightarrow \mathrm{B} 3 \rightarrow \mathrm{B} 2 \rightarrow \mathrm{B} 1$ na presença da cor vermelha. Um participante $(\mathrm{GV})$ precisou de oito exposições à seqüência $\mathrm{A} 1 \rightarrow \mathrm{A} 2 \rightarrow \mathrm{A} 3 \rightarrow \mathrm{A} 4 \rightarrow \mathrm{A} 5 \rightarrow \mathrm{A} 6$ na presença da cor verde e seis exposições na seqüência inversa $\mathrm{A} 6 \rightarrow \mathrm{A} 5 \rightarrow \mathrm{A} 4 \rightarrow \mathrm{A} 3 \rightarrow \mathrm{A} 2 \rightarrow$ A1 na presença da cor vermelha. Um outro participante (WS) precisou de nove exposições ao treino na seqüêencia $\mathrm{B} 1 \rightarrow$ $\mathrm{B} 2 \rightarrow \mathrm{B} 3 \rightarrow \mathrm{B} 4 \rightarrow \mathrm{B} 5 \rightarrow \mathrm{B} 6$ na presença da cor verde, oito vezes na seqüência $\mathrm{B} 6 \rightarrow \mathrm{B} 5 \rightarrow \mathrm{B} 4 \rightarrow \mathrm{B} 3 \rightarrow \mathrm{B} 2 \rightarrow \mathrm{B} 1$ na presença da cor vermelha e sete vezes na seqüência $\mathrm{C} 6 \rightarrow \mathrm{C} 5 \rightarrow \mathrm{C} 4 \rightarrow \mathrm{C} 3 \rightarrow$ $\mathrm{C} 2 \rightarrow \mathrm{C} 1$ na presença da cor vermelha.

A Tabela 6 a seguir apresenta o resultado dos participantes nos testes de seqüênciação. Todos responderam prontamente. O participante (EG) respondeu corretamente com atraso.

A Tabela 7 apresenta o resultado dos participantes nos testes de transitividade na presença da cor verde. Todos responderam prontamente, exceto (GV, EG e LP) que precisaram de mais uma exposição à tentativa, ou seja, responderam com atraso. 
Tabela 5. Desempenho dos participantes em cada tentativa de treino por encadeamento, na presença da cor verde ou vermelha.

\begin{tabular}{|c|c|c|c|c|c|c|}
\hline \multicolumn{2}{|c|}{ Tipo de Tentativa } & \multicolumn{5}{|c|}{ Número de tentativas por participantes } \\
\hline \multicolumn{2}{|c|}{ Treino de Encadeamento Cores } & WS & DP & GV & EG & $\mathbf{L P}$ \\
\hline $\mathrm{A} 1 \rightarrow \mathrm{A} 2 \rightarrow \mathrm{A} 3 \rightarrow \mathrm{A} 4 \rightarrow \mathrm{A} 5 \rightarrow \mathrm{A} 6$ & Verde & * & $*$ & $* * * *$ & $*$ & $*$ \\
\hline $\mathrm{A} 6 \rightarrow \mathrm{A} 5 \rightarrow \mathrm{A} 4 \rightarrow \mathrm{A} 3 \rightarrow \mathrm{A} 2 \rightarrow \mathrm{A} 1$ & Vermelha & $*$ & $*$ & $* *$ & $*$ & $*$ \\
\hline $\mathrm{B} 1 \rightarrow \mathrm{B} 2 \rightarrow \mathrm{B} 3 \rightarrow \mathrm{B} 4 \rightarrow \mathrm{B} 5 \rightarrow \mathrm{B} 6$ & Verde & $* * * * *$ & $* * *$ & $*$ & $*$ & $*$ \\
\hline $\mathrm{B} 6 \rightarrow \mathrm{B} 5 \rightarrow \mathrm{B} 4 \rightarrow \mathrm{B} 3 \rightarrow \mathrm{B} 2 \rightarrow \mathrm{B} 1$ & Vermelha & $* * * *$ & $* * * *$ & $*$ & $*$ & $*$ \\
\hline $\mathrm{C} 1 \rightarrow \mathrm{C} 2 \rightarrow \mathrm{C} 3 \rightarrow \mathrm{C} 4 \rightarrow \mathrm{C} 5 \rightarrow \mathrm{C} 6$ & Verde & $*$ & $*$ & $*$ & $*$ & $*$ \\
\hline $\mathrm{C} 6 \rightarrow \mathrm{C} 5 \rightarrow \mathrm{C} 4 \rightarrow \mathrm{C} 3 \rightarrow \mathrm{C} 2 \rightarrow \mathrm{C} 1$ & Vermelha & $* * *$ & $*$ & $*$ & $*$ & $*$ \\
\hline
\end{tabular}

* Participante alcançou critério de acerto

** Participante foi exposto a seis tentativas

*** Participante foi exposto sete vezes à tentativa

**** Participante foi exposto oito vezes à tentativa

***** Participante foi exposto nove vezes à tentativa

Tabela 6. Desempenho dos participantes em cada tentativa de teste de seqüênciação, na presença da cor verde ou vermelha.

\begin{tabular}{|c|c|c|c|c|c|c|}
\hline \multirow{2}{*}{\multicolumn{2}{|c|}{ Testes de Seqüenciação Cores }} & \multicolumn{5}{|c|}{ Número de tentativas por participantes } \\
\hline & & \multirow{2}{*}{$\begin{array}{c}\text { WS } \\
*\end{array}$} & \multirow{2}{*}{$\frac{\text { DP }}{*}$} & \multirow{2}{*}{$\frac{\mathbf{G V}}{*}$} & \multirow{2}{*}{$\begin{array}{c}\mathbf{E G} \\
* *\end{array}$} & \multirow{2}{*}{$\frac{\mathbf{L P}}{*}$} \\
\hline $\mathrm{A} 1 \rightarrow \mathrm{A} 2 \rightarrow \mathrm{A} 3 \rightarrow \mathrm{A} 4 \rightarrow \mathrm{A} 5 \rightarrow \mathrm{A} 6$ & Verde & & & & & \\
\hline $\mathrm{A} 6 \rightarrow \mathrm{A} 5 \rightarrow \mathrm{A} 4 \rightarrow \mathrm{A} 3 \rightarrow \mathrm{A} 2 \rightarrow \mathrm{A} 1$ & Vermelha & $*$ & $*$ & * & $* *$ & $*$ \\
\hline $\mathrm{B} 1 \rightarrow \mathrm{B} 2 \rightarrow \mathrm{B} 3 \rightarrow \mathrm{B} 4 \rightarrow \mathrm{B} 5 \rightarrow \mathrm{B} 6$ & Verde & $*$ & $*$ & $*$ & $*$ & $*$ \\
\hline $\mathrm{B} 6 \rightarrow \mathrm{B} 5 \rightarrow \mathrm{B} 4 \rightarrow \mathrm{B} 3 \rightarrow \mathrm{B} 2 \rightarrow \mathrm{B} 1$ & Vermelha & $*$ & $*$ & $*$ & $* *$ & $*$ \\
\hline $\mathrm{C} 1 \rightarrow \mathrm{C} 2 \rightarrow \mathrm{C} 3 \rightarrow \mathrm{C} 4 \rightarrow \mathrm{C} 5 \rightarrow \mathrm{C} 6$ & Verde & $*$ & $*$ & * & $*$ & $*$ \\
\hline $\mathrm{C} 6 \rightarrow \mathrm{C} 5 \rightarrow \mathrm{C} 4 \rightarrow \mathrm{C} 3 \rightarrow \mathrm{C} 2 \rightarrow \mathrm{C} 1$ & Vermelha & $*$ & $*$ & * & $*$ & $*$ \\
\hline
\end{tabular}

* Participante respondeu prontamente.

** Participante respondeu com atraso

A Tabela 8 apresenta o resultado dos participantes nos testes de transitividade na presença da cor vermelha. Todos responderam prontamente, exceto (WS e DP) que precisaram de mais uma exposição às tentativas.

A Tabela 9 apresenta o resultado dos participantes na revisão de linha de base na presença das cores verde e vermelho. Todos alcançaram o critério de acerto e em seguida eram expostos aos testes de conectividade.

A Tabela 10 apresenta o resultado dos participantes nos testes de conectividade na presença das cores verde e vermelho. Todos responderam corretamente e na primeira tentativa, ou seja, prontamente, embora precisassem em algumas seqüências de uma re-exposição, ou seja, responderam corretamente com atraso.

\section{Discussão}

Todos os participantes alcançaram o critério de acerto e responderam prontamente ou com atraso, os testes de transitividade e de conectividade. $\mathrm{O}$ ensino de três sequiências de respostas independentes na presença das cores verde e vermelho levou os participantes, após alcançarem o critério de acerto na linha de base, a responderem condicionalmente, apresentando 12 novas sequiências de respostas prontamente sem qualquer treino adicional, demonstrando com isso a emergência de classes ordinais com seis estímulos. Novas seqüências poderiam ter sido estabelecidas sem qualquer treino adicional, por exemplo: $\mathrm{A} 1 \rightarrow \mathrm{B} 2 \rightarrow \mathrm{C} 3 \rightarrow \mathrm{A} 4 \rightarrow \mathrm{B} 5 \rightarrow$ $\mathrm{C} 6$ na presença da cor verde ou $\mathrm{C} 6 \rightarrow \mathrm{B} 5 \rightarrow \mathrm{A} 4 \rightarrow \mathrm{C} 3 \rightarrow \mathrm{B} 2 \rightarrow$ $\mathrm{A} 1$ na presença da cor vermelha, ou $\mathrm{C} 1 \rightarrow \mathrm{A} 2 \rightarrow \mathrm{B} 3 \rightarrow \mathrm{C} 4 \rightarrow$ $\mathrm{A} 5 \rightarrow \mathrm{B} 6$ na presença da cor verde ou $\mathrm{B} 6 \rightarrow \mathrm{A} 5 \rightarrow \mathrm{C} 4 \rightarrow \mathrm{B} 3 \rightarrow$ $\mathrm{A} 2 \rightarrow \mathrm{C} 1$ na presença da cor vermelha, ampliando as possibilidades de novas relações ordinais. Essa produtividade do comportamento é coerente com o paradigma de equivalência proposta por Sidman $(1986,1994)$.

Em relação ao atraso na emergência de relações de equivalência, Green e Saunders (1998) afirmaram que " $a$ emergência gradual durante os testes pode ser influenciada pela ordem de apresentação dos mesmos" (p. 253). O contato do participante com a contingência de teste foi adiada após longas tentativas de treino. Isto pode ter contribuído para essa emergência gradual de algumas relações ordinais. No presente estudo exigiu-se uma resposta de observação ao apontar inicialmente para a cor no alto da tela. Uma questão empírica que precisa ser mais bem investigada é se emergiriam sequiências de respostas distintas se, por exemplo, topografias de respostas diferentes (verbais ou não) fossem programadas na presença dessas cores. 
Tabela 7. Desempenho dos participantes nos testes de transitividade, na presença da cor verde.

\begin{tabular}{|c|c|c|c|c|c|}
\hline $\begin{array}{l}\text { Tipo de tentativa nos } \\
\text { testes de transitividade }\end{array}$ & WS & DP & GV & EG & LP \\
\hline $\mathrm{A} 1 \rightarrow \mathrm{A} 3$ & $*$ & $*$ & $*$ & $*$ & $*$ \\
\hline $\mathrm{A} 1 \rightarrow \mathrm{A} 4$ & $*$ & $*$ & $*$ & $*$ & $*$ \\
\hline $\mathrm{A} 1 \rightarrow \mathrm{A} 5$ & $*$ & $*$ & $*$ & $*$ & $*$ \\
\hline $\mathrm{A} 1 \rightarrow \mathrm{A} 6$ & $*$ & $*$ & $*$ & $*$ & $*$ \\
\hline $\mathrm{A} 2 \rightarrow \mathrm{A} 4$ & $*$ & $*$ & $*$ & $*$ & $*$ \\
\hline $\mathrm{A} 2 \rightarrow \mathrm{A} 5$ & $*$ & $*$ & $*$ & $*$ & $*$ \\
\hline $\mathrm{A} 2 \rightarrow \mathrm{A} 6$ & $*$ & $*$ & $*$ & $*$ & $*$ \\
\hline $\mathrm{A} 3 \rightarrow \mathrm{A} 5$ & $*$ & $*$ & $*$ & $*$ & $*$ \\
\hline $\mathrm{A} 3 \rightarrow \mathrm{A} 6$ & $*$ & $*$ & $* *$ & $*$ & $*$ \\
\hline $\mathrm{A} 4 \rightarrow \mathrm{A} 6$ & $*$ & $*$ & $*$ & $* *$ & $*$ \\
\hline $\mathrm{B} 1 \rightarrow \mathrm{B} 3$ & $*$ & $*$ & $*$ & $*$ & $*$ \\
\hline $\mathrm{B} 1 \rightarrow \mathrm{B} 4$ & $*$ & $*$ & $*$ & $*$ & $*$ \\
\hline $\mathrm{B} 1 \rightarrow \mathrm{B} 5$ & $*$ & $*$ & $*$ & $*$ & $*$ \\
\hline $\mathrm{B} 1 \rightarrow \mathrm{B} 6$ & $*$ & $*$ & $*$ & $*$ & $*$ \\
\hline $\mathrm{B} 2 \rightarrow \mathrm{B} 4$ & $*$ & $*$ & $*$ & $*$ & $*$ \\
\hline $\mathrm{B} 2 \rightarrow \mathrm{B} 5$ & $*$ & $*$ & $*$ & $*$ & $*$ \\
\hline $\mathrm{B} 2 \rightarrow \mathrm{B} 6$ & $*$ & $*$ & $*$ & $*$ & $*$ \\
\hline $\mathrm{B} 3 \rightarrow \mathrm{B} 5$ & $*$ & $*$ & $*$ & $*$ & $*$ \\
\hline $\mathrm{B} 3 \rightarrow \mathrm{B} 6$ & $*$ & $*$ & $*$ & $*$ & $*$ \\
\hline $\mathrm{B} 4 \rightarrow \mathrm{B} 6$ & $*$ & $*$ & $*$ & $*$ & $* *$ \\
\hline $\mathrm{C} 1 \rightarrow \mathrm{C} 3$ & $*$ & $*$ & $*$ & $*$ & $*$ \\
\hline $\mathrm{C} 1 \rightarrow \mathrm{C} 4$ & $*$ & $*$ & $*$ & $*$ & $*$ \\
\hline $\mathrm{C} 1 \rightarrow \mathrm{C} 5$ & $*$ & $*$ & $*$ & $*$ & $*$ \\
\hline $\mathrm{C} 1 \rightarrow \mathrm{C} 6$ & $*$ & $*$ & $*$ & $*$ & $*$ \\
\hline $\mathrm{C} 2 \rightarrow \mathrm{C} 4$ & $*$ & $*$ & $*$ & $*$ & $*$ \\
\hline $\mathrm{C} 2 \rightarrow \mathrm{C} 5$ & $*$ & $*$ & $*$ & $*$ & $*$ \\
\hline $\mathrm{C} 2 \rightarrow \mathrm{C} 6$ & $*$ & $*$ & $*$ & $*$ & $*$ \\
\hline $\mathrm{C} 3 \rightarrow \mathrm{C} 5$ & $*$ & $*$ & $*$ & $*$ & $*$ \\
\hline $\mathrm{C} 3 \rightarrow \mathrm{C} 6$ & $*$ & $*$ & $*$ & $*$ & $*$ \\
\hline $\mathrm{C} 4 \rightarrow \mathrm{C} 6$ & $*$ & $*$ & $*$ & $*$ & $*$ \\
\hline
\end{tabular}

*Participante respondeu prontamente.

**Participante respondeu com atraso

Outra interpretação relevante desses resultados estaria na análise do comportamento de contar. Ou seja, os resultados do presente estudo parecem confirmar as hipóteses de alguns autores (Carmo, 1997; Green, 1993) que apontam a não necessidade do ensino prévio da contagem para a aquisição de relações numéricas. Os participantes desse primeiro estudo eram alunos em fase de alfabetização e pré-testes avaliaram que os mesmos não apresentavam repertórios matemáticos. Portanto, a ordinalidade ocorreu a partir dessa rede de relações entre estímulos equivalentes.

Esses resultados replicaram sistematicamente e estendem os observados na literatura com o procedimento de ensino por encadeamento sob controle condicional (Assis \& Costa, 2004; Lazar \& Kotlarchyk, 1986; Stromer \& Mackay, 1992), sugerindo que esse tipo de treino foi eficiente na emergência de classes ordinais. Uma questão experimental ainda a ser investigada em estudos posteriores seria avaliar se esse responder seqüencial se manteria estável em contingências com cinco termos (controle contextual).
Tabela 8. Desempenho dos participantes nos testes de transitividade, na presença da cor vermelha.

\begin{tabular}{|c|c|c|c|c|c|}
\hline $\begin{array}{l}\text { Tipo de tentativa nos } \\
\text { testes de transitividade }\end{array}$ & WS & DP & GV & EG & $\mathbf{L P}$ \\
\hline $\mathrm{A} 6 \rightarrow \mathrm{A} 4$ & $*$ & $*$ & $*$ & $*$ & $*$ \\
\hline $\mathrm{A} 6 \rightarrow \mathrm{A} 3$ & $*$ & $*$ & $*$ & $*$ & $*$ \\
\hline $\mathrm{A} 6 \rightarrow \mathrm{A} 2$ & $*$ & $*$ & $*$ & $*$ & $*$ \\
\hline $\mathrm{A} 6 \rightarrow \mathrm{A} 1$ & $*$ & $*$ & $*$ & $*$ & $*$ \\
\hline $\mathrm{A} 5 \rightarrow \mathrm{A} 3$ & $*$ & $*$ & $*$ & $*$ & $*$ \\
\hline $\mathrm{A} 5 \rightarrow \mathrm{A} 2$ & $*$ & $*$ & $*$ & $*$ & $*$ \\
\hline $\mathrm{A} 5 \rightarrow \mathrm{A} 1$ & $* *$ & $*$ & $*$ & $*$ & $*$ \\
\hline $\mathrm{A} 4 \rightarrow \mathrm{A} 2$ & $*$ & $*$ & $*$ & $*$ & $*$ \\
\hline $\mathrm{A} 4 \rightarrow \mathrm{A} 1$ & $*$ & $*$ & $*$ & $*$ & $*$ \\
\hline $\mathrm{A} 3 \rightarrow \mathrm{A} 1$ & $*$ & $*$ & $*$ & $*$ & $*$ \\
\hline $\mathrm{B} 6 \rightarrow \mathrm{B} 4$ & $*$ & $*$ & * & * & $*$ \\
\hline $\mathrm{B} 6 \rightarrow \mathrm{B} 3$ & $*$ & $*$ & $*$ & $*$ & $*$ \\
\hline $\mathrm{B} 6 \rightarrow \mathrm{B} 2$ & $*$ & $*$ & $*$ & $*$ & $*$ \\
\hline $\mathrm{B} 6 \rightarrow \mathrm{B} 1$ & $*$ & $*$ & $*$ & $*$ & $*$ \\
\hline $\mathrm{B} 5 \rightarrow \mathrm{B} 3$ & $*$ & $*$ & $*$ & $*$ & $*$ \\
\hline $\mathrm{B} 5 \rightarrow \mathrm{B} 2$ & $*$ & $*$ & $*$ & $*$ & $*$ \\
\hline $\mathrm{B} 5 \rightarrow \mathrm{B} 1$ & $*$ & $*$ & $*$ & $*$ & $*$ \\
\hline $\mathrm{B} 4 \rightarrow \mathrm{B} 2$ & $*$ & $*$ & $*$ & $*$ & $*$ \\
\hline $\mathrm{B} 4 \rightarrow \mathrm{B} 1$ & $*$ & $*$ & $*$ & $*$ & $*$ \\
\hline $\mathrm{B} 3 \rightarrow \mathrm{B} 1$ & $*$ & $* *$ & $*$ & $*$ & $*$ \\
\hline $\mathrm{C} 6 \rightarrow \mathrm{C} 4$ & $*$ & $*$ & $*$ & $*$ & $*$ \\
\hline $\mathrm{C} 6 \rightarrow \mathrm{C} 3$ & $*$ & $*$ & $*$ & $*$ & $*$ \\
\hline $\mathrm{C} 6 \rightarrow \mathrm{C} 2$ & $*$ & $*$ & $*$ & $*$ & $*$ \\
\hline $\mathrm{C} 6 \rightarrow \mathrm{C} 1$ & $*$ & $*$ & $*$ & $*$ & $*$ \\
\hline $\mathrm{C} 5 \rightarrow \mathrm{C} 3$ & $*$ & $*$ & $*$ & $*$ & $*$ \\
\hline $\mathrm{C} 5 \rightarrow \mathrm{C} 2$ & $*$ & $*$ & $*$ & $*$ & $*$ \\
\hline $\mathrm{C} 5 \rightarrow \mathrm{C} 1$ & $*$ & $*$ & $*$ & $*$ & $*$ \\
\hline $\mathrm{C} 4 \rightarrow \mathrm{C} 2$ & $*$ & $*$ & $*$ & $*$ & $*$ \\
\hline $\mathrm{C} 4 \rightarrow \mathrm{C} 1$ & $*$ & $*$ & $*$ & $*$ & $*$ \\
\hline $\mathrm{C} 3 \rightarrow \mathrm{C} 1$ & $*$ & $*$ & $*$ & $*$ & $*$ \\
\hline
\end{tabular}

*Participante respondeu prontamente.

** Participante respondeu com atraso

Os dados apresentados pela literatura nesta linha de pesquisa, assim como os resultados descritos neste estudo, apontam para a emergência de classes ordinais, a partir de pelo menos duas sequiências de respostas ensinadas independentemente, sem qualquer treino adicional. Desse modo, como explicar o ordenar na ausência de um estímulo que exerceria uma dupla função (reforçadora condicionada e discriminativa) numa cadeia comportamental? Catania (1998/1999) tem proposto que poderíamos analisar comportamento seqüencial enquanto "unidades de comportamento temporalmente estendidas, não redutíveis a tais seqüências" (p.143) para se contrapor à concepção de sequiências de estímulo-resposta. Portanto, ordenar um conjunto de estímulos com seis elementos dispostos simultaneamente, constituiria um operante, na medida que somente a última resposta é reforçada diferencialmente. Essas classes de estímulos eram estabelecidas a partir da mediação de uma resposta comum, ou seja, a seleção dos estímulos em uma determinada ordem. 
Tabela 9. Desempenho dos participantes em cada tentativa da revisão de linha de base por participante, na presença da cor verde ou vermelha.

\begin{tabular}{|c|c|c|c|c|c|c|}
\hline \multirow{2}{*}{\multicolumn{2}{|c|}{ Tipos de tentativas na linha de base nas cores verde e vermelho }} & \multicolumn{5}{|c|}{ Número de tentativas por participantes } \\
\hline & & \multirow{2}{*}{$\begin{array}{c}\text { WS } \\
*\end{array}$} & \multirow{2}{*}{$\begin{array}{c}\text { DP } \\
*\end{array}$} & \multirow{2}{*}{$\frac{\mathbf{G V}}{*}$} & \multirow{2}{*}{$\begin{array}{c}\mathbf{E G} \\
*\end{array}$} & \multirow{2}{*}{$\begin{array}{r}\mathbf{L P} \\
*\end{array}$} \\
\hline $\mathrm{A} 1 \rightarrow \mathrm{A} 2 \rightarrow \mathrm{A} 3 \rightarrow \mathrm{A} 4 \rightarrow \mathrm{A} 5 \rightarrow \mathrm{A} 6$ & Verde & & & & & \\
\hline $\mathrm{A} 6 \rightarrow \mathrm{A} 5 \rightarrow \mathrm{A} 4 \rightarrow \mathrm{A} 3 \rightarrow \mathrm{A} 2 \rightarrow \mathrm{A} 1$ & Vermelha & $*$ & $*$ & $*$ & $*$ & $*$ \\
\hline $\mathrm{B} 1 \rightarrow \mathrm{B} 2 \rightarrow \mathrm{B} 3 \rightarrow \mathrm{B} 4 \rightarrow \mathrm{B} 5 \rightarrow \mathrm{B} 6$ & Verde & $*$ & $*$ & $*$ & $*$ & $*$ \\
\hline $\mathrm{B} 6 \rightarrow \mathrm{B} 5 \rightarrow \mathrm{B} 4 \rightarrow \mathrm{B} 3 \rightarrow \mathrm{B} 2 \rightarrow \mathrm{B} 1$ & Vermelha & $*$ & $*$ & $*$ & $*$ & * \\
\hline $\mathrm{C} 1 \rightarrow \mathrm{C} 2 \rightarrow \mathrm{C} 3 \rightarrow \mathrm{C} 4 \rightarrow \mathrm{C} 5 \rightarrow \mathrm{C} 6$ & Verde & $*$ & $*$ & $*$ & $*$ & $*$ \\
\hline $\mathrm{C} 6 \rightarrow \mathrm{C} 5 \rightarrow \mathrm{C} 4 \rightarrow \mathrm{C} 3 \rightarrow \mathrm{C} 2 \rightarrow \mathrm{C} 1$ & Vermelha & $*$ & $*$ & $*$ & $*$ & $*$ \\
\hline
\end{tabular}

* Participante alcançou critério de acerto

Tabela 10. Desempenho dos participantes em cada tentativa nos testes de conectividade, na presença da cor verde ou vermelha.

\begin{tabular}{|c|c|c|c|c|c|c|}
\hline \multirow{2}{*}{ Teste de conectividade } & \multirow{2}{*}{ Cores } & \multicolumn{5}{|c|}{ Número de tentativas por participantes } \\
\hline & & WS & DP & GV & EG & $\mathbf{L P}$ \\
\hline $\mathrm{A} 1 \rightarrow \mathrm{B} 2 \rightarrow \mathrm{A} 3 \rightarrow \mathrm{B} 4 \rightarrow \mathrm{A} 5 \rightarrow \mathrm{B} 6$ & Verde & $*$ & $*$ & $*$ & $*$ & $*$ \\
\hline $\mathrm{A} 6 \rightarrow \mathrm{B} 5 \rightarrow \mathrm{A} 4 \rightarrow \mathrm{B} 3 \rightarrow \mathrm{A} 2 \rightarrow \mathrm{B} 1$ & Vermelho & $*$ & $*$ & $*$ & $* *$ & $*$ \\
\hline $\mathrm{B} 1 \rightarrow \mathrm{A} 2 \rightarrow \mathrm{B} 3 \rightarrow \mathrm{A} 4 \rightarrow \mathrm{B} 5 \rightarrow \mathrm{A} 6$ & Verde & $*$ & $* *$ & $*$ & $* *$ & $*$ \\
\hline $\mathrm{B} 6 \rightarrow \mathrm{A} 5 \rightarrow \mathrm{B} 4 \rightarrow \mathrm{A} 3 \rightarrow \mathrm{B} 2 \rightarrow \mathrm{A} 1$ & Vermelho & $*$ & * & $*$ & $*$ & $*$ \\
\hline $\mathrm{A} 1 \rightarrow \mathrm{C} 2 \rightarrow \mathrm{A} 3 \rightarrow \mathrm{C} 4 \rightarrow \mathrm{A} 5 \rightarrow \mathrm{C} 6$ & Verde & $*$ & * & $*$ & $*$ & $*$ \\
\hline $\mathrm{A} 6 \rightarrow \mathrm{C} 5 \rightarrow \mathrm{A} 4 \rightarrow \mathrm{C} 3 \rightarrow \mathrm{A} 2 \rightarrow \mathrm{C} 1$ & Vermelho & $* *$ & * & $*$ & $* *$ & $*$ \\
\hline $\mathrm{C} 1 \rightarrow \mathrm{A} 2 \rightarrow \mathrm{C} 3 \rightarrow \mathrm{A} 4 \rightarrow \mathrm{C} 5 \rightarrow \mathrm{A} 6$ & Verde & $*$ & $*$ & $*$ & $*$ & $*$ \\
\hline $\mathrm{C} 6 \rightarrow \mathrm{A} 5 \rightarrow \mathrm{C} 4 \rightarrow \mathrm{A} 3 \rightarrow \mathrm{C} 2 \rightarrow \mathrm{A} 1$ & Vermelho & $*$ & $* *$ & $*$ & $* *$ & $* *$ \\
\hline $\mathrm{B} 1 \rightarrow \mathrm{C} 2 \rightarrow \mathrm{B} 3 \rightarrow \mathrm{C} 4 \rightarrow \mathrm{B} 5 \rightarrow \mathrm{C} 6$ & Verde & $*$ & $*$ & $*$ & $*$ & $*$ \\
\hline $\mathrm{B} 6 \rightarrow \mathrm{C} 5 \rightarrow \mathrm{B} 4 \rightarrow \mathrm{C} 3 \rightarrow \mathrm{B} 2 \rightarrow \mathrm{C} 1$ & Vermelho & $*$ & $*$ & $* *$ & $*$ & $*$ \\
\hline $\mathrm{C} 1 \rightarrow \mathrm{B} 2 \rightarrow \mathrm{C} 3 \rightarrow \mathrm{B} 4 \rightarrow \mathrm{C} 5 \rightarrow \mathrm{B} 6$ & Verde & $* *$ & $*$ & $*$ & $* *$ & $*$ \\
\hline $\mathrm{C} 6 \rightarrow \mathrm{B} 5 \rightarrow \mathrm{C} 4 \rightarrow \mathrm{B} 3 \rightarrow \mathrm{C} 2 \rightarrow \mathrm{B} 1$ & Vermelho & $*$ & $*$ & $*$ & $* *$ & $*$ \\
\hline
\end{tabular}

* Participante respondeu prontamente.

** Participante respondeu com atraso

Classes ordinais emergem a partir dessa intercambialidade funcional derivada das contingências de treino que o participante tenha sido exposto. Entretanto, como afirma Sidman (1997): "Contingências de reforçamento não cria relações de equivalência; elas estabelecem os pré-requisitos para a demonstração das propriedades que definem uma relação de equivalência" (p. 259). Desse modo, não precisamos recorrer a conceitos de representação, idéias, raciocínio ou mapas cognitivos para explicar esses novos desempenhos. Isto também parece coerente com a revisão do paradigma de equivalência (cf. Sidman, 1994; 2000) que incorpora a resposta e o reforço na definição de equivalência, removendo a distinção entre estímulos e respostas na concepção de união de classes. Essa questão foi levantada por Mackay e cols. (1998) quando discutem o termo "transferência de funções" na substitutabilidade de estímulos enquanto fusão de classes arbitrárias. Ou seja, na emergência de novas sequiências pela substitutabilidade de estímulos, qual a natureza do controle de estímulos envolvidos na seqüência? O conceito de encadeamento (Skinner, 1938) parece que não daria conta de explicitá-lo.

Alguns autores (McIlvane, Serna, Dube \& Stromer, 2000) ainda têm chamado atenção para a coerência do que é efetivamente programado com o responder do participante. Ou seja, garantir que as contingências de testes sejam semelhantes às contingências de treino reduz a probabilidade de erros, assegurando resultados positivos em testes de conectividade. Testes adicionais precisariam ser conduzidos para avaliar com mais segurança a estabilidade das classes ordinais.

A aplicação de testes de generalização com novos estímulos não foi realizada no presente estudo, sendo um dado importante a ser explorado em futuras pesquisas. Isso poderia avaliar se crianças com surdez teriam dificuldades para identificar e nomear novos estímulos. Testes finais de nomeação (por exemplo, perguntar ao participante, quantos elementos têm neste conjunto de figuras?) poderiam explicitar ainda mais o tipo de controle exercido por propriedades relacionais (Catania, 1998/1999) como estas descritas neste estudo.

A demonstração do controle condicional sobre o responder seqüencial tem importantes implicações acadêmicas no campo da leitura e da escrita (por exemplo, na cultura ocidental, a redação e leitura de uma sentença são da esquerda para a direita, diferentemente da cultura japonesa). Além disso, pequenas mudanças de posição de uma palavra ou de um sinal gráfico na frase, podem alterar o significado da sentença. Entretanto, nas operações fundamentais da matemática, a ordem dos fatores não altera o produto. Do ponto de vista comportamental, ordenar do menor para o maior ou vice-versa estaria simplesmente sob controle da numerosidade enquanto uma propriedade das relações entre estímulos. 


\section{Referências}

Assis, G. J. A. \& Costa, L. C. A. (2004). Busca de relações ordinais em crianças. Revista Interação em Psicologia, 8(2), 199-216.

Carmo, J. S. (1997). Aquisição do conceito de número em crianças pré-escolares através do ensino de relações condicionais e generalização. Dissertação de Mestrado, Universidade Federal do Pará, Belém.

Carr, D., Wilkinson, K. M., Blackman, D. \& McIlvane, W. J. (2000). Equivalence classes in individuals with minimal verbal repertoires. Journal of the Experimental Analysis of Behavior, 74, 101-114.

Catania, A. C. (1999). Aprendizagem: comportamento, linguageme cognição. (D. G. Souza, Trad.) Porto Alegre: Artes Médicas.

Green, G. (1993). Stimulus control technology for teaching number/ quantity equivalences [Completo]. Em Proceedings. Melbourne: Victoria Autistic Children's \& Adults' Association (Org.), The Conference of The National Association For Autism (51-63), Melbourne.

Green, G., Stromer, R. \& Mackay, H. (1993). Relational learning in stimulus sequences. The_Psychological Record, 43, 599-616.

Green, G. \& Saunders, R. (1998). Stimulus equivalence. Em K. A. Lattal \& M. Perone (Orgs.), Handbook of Research Methods in Human Operant Behavior (pp.229-262). New York: Plenum Press.

Kahhale, E. M. S. P. (1993). Comportamento matemático: formação e ampliação do conceito de quantidade e relações de equivalência. Tese de Doutorado, Universidade de São Paulo, São Paulo.

Lazar, R. \& Kotlarchyk, B. J. (1986). Second order control of sequence class equivalence in children. Behavioral Process. 13, 205-215.

Mackay, H. A., Kotlarchyk, B. J. \& Stromer, R. (1997). Stimulus classes, stimulus sequences, and generative behavior. Em D. Baer \& E. M. Pinkston (Orgs.), Environment and Behavior (pp. 124-137). Boulder, CO: WestviewPress.

Mackay, H. A., Stromer, R. \& Serna, R. W. (1998). Emergent behavior and intellectual functioning: stimulus classes, generalization, and transfer. Em S. Soraci \& W. J. McIlvane (Orgs.), Perspectives on Fundamental Processes in Intellectual Functioning (pp.287-310). Norwood, NJ: Ablex.

Maydak, M., Stromer, R., Mackay, H. \& Stoddard, L. (1995). Stimulus classes in matching to sample and sequence production: the emergence of numeric relations. Research in Developmental Disabilities, 16, 179-204.
McIlvane, W. J., Serna, R.W., Dube, W. V. \& Stromer, R. (2000). Stimulus control topography coherence and stimulus equivalence: reconciling test outcomes with theory. Em J. C. Leslie \& D. Blackman (Orgs.), Experimental and Applied Analysis of Human Behavior (pp. 85-110). Reno, NV: Context Press.

Prado, P. S. T. (1995). O conceito de número: uma análise na perspectiva do paradigma de rede de relações. Dissertação de Mestrado, Universidade Federal de São Carlos, São Carlos.

Prado, P. S. T. (2001). Ensinando o conceito de número: contribuições do paradigma de rede de relações. Tese de Doutorado, Universidade de São Paulo, São Paulo.

Prado, P. S. T. \& de Rose, J. C. C. (1999). Conceito de número: uma contribuição da análise do comportamento da cognição. Psicologia: Teoria e Pesquisa, 15(3), 227-235.

Sidman. M. (1986). Functional Analysis of Emergent Verbal Classes. Em T. Thompson \& M. D. L. Zeiler (Orgs.). Analysis and Integration of Behavioral Units (p.213-245). Hillsdale, N. J.: Lawrence Erlbaum Associates Publishers.

Sidman. M. (1994). Equivalence Relations and Behavior: A Research Story. Boston: Authors Cooperative, Inc. Publishers.

Sidman, M. (1997). Equivalence relations. Journal of the Experimental Analysis of Behavior, 68, 258-266.

Sidman, M. (2000). Equivalence relations and the reinforcement contingency. Journal of the Experimental Analysis of Behavior, 74, 127-146.

Sidman, M. \& Tailby, W. (1982). Conditional discrimination vs matching to sample: an expansion of the testing paradigm. Journal of the Experimental Analysis of Behavior, 37, 5-22.

Stevens, S. S. (1951). Mathematics, Measurement, and Psychophysics. Em S. S. Stevens (Org.), Handbook of Experimental Psychology (pp.1-49). New York: John Wiley and Sons, Inc.

Stromer, R. \& Mackay, H. A (1992). Conditional stimulus control of children's sequence production. Psychological Reports, 70, 903-912.

Skinner, B. F. (1938). The Behavior of Organisms. New York: Appleton-Century-Crofts.

Williams, K. D. (2000). Teaching pre-math skills via stimulus equivalence procedures. Master Thesis. Southern Illinois University at Carbondale.

Yamamoto, J. (1994). Functional Analysis of Verbal Behavior in Handicapped Children. Em S. Hayes, L. Hayes, M. Sato \& K. Ono (Orgs.), Behavior Analysis of Language and Cognition (pp.107-122). Reno, NV: Context Press. 